\title{
Activation of Autophagy Protects Against ROS-Mediated Mitochondria-Dependent Apoptosis in L-02 Hepatocytes Induced by $\operatorname{Cr}(\mathrm{VI})$
}

\author{
Ying Xie Fang Xiao Lei Luo Caigao Zhong \\ Department of Health Toxicology, School of Public Health, Central South University, Changsha, \\ People's Republic of China
}

\author{
Key Words \\ Autophagy $\bullet$ Hepatocytes $\bullet$ ROS $・$ Mitochondria $\cdot$ Apoptosis
}

\begin{abstract}
Background: Hexavalent chromium ( $\mathrm{Cr}(\mathrm{VI})$ ) overdose causes hepatocellular injuries by inducing mitochondrial damage and subsequent apoptosis in animals and humans. Autophagy can selectively remove damaged organelles, especially impaired mitochondria, and in turn, protects against mitochondria-dependent cell death. The present study was designed to explore the effects of autophagy on the $\mathrm{Cr}(\mathrm{VI})$-induced hepatotoxicity. Methods: $\mathrm{L}-02$ hepatocytes were incubated with different concentrations of $\mathrm{Cr}(\mathrm{VI})$ for $24 \mathrm{~h}$ and several indicators for evaluating mitochondrial damage and hepatocellular apoptosis were measured. Then effects of ROS scavenger NAC on ROS production and calcium overload during $\mathrm{Cr}(\mathrm{VI})-$ induced hepatotoxicity were examined. Finally, the study further investigated the role of autophagy played in repairing mitochondrial damage and subsequent hepatocyte injuries. Results: After exposed to different concentrations of $\mathrm{Cr}(\mathrm{VI})$ for $24 \mathrm{~h}$, cell viability, mitochondria membrane potential, ATP content were significantly decreased and caspase- 3 activities and apoptosis rates increased in L-02 hepatocytes. The treatment of NAC reduced ROS formation and $\mathrm{Ca}^{2+}$ content, restored CRAC channel activities and further diminished mitochondrial injuries. Furthermore, autophagy inducer, rapamycin is beneficial for repairing mitochondrial function and limiting hepatocytes damage, and pharmacological inhibition of autophagy by 3-methyladenine further exacerbated $\mathrm{Cr}(\mathrm{VI})$-induced hepatotoxicity. Conclusions: ROS production is a critical reason for $\mathrm{Cr}(\mathrm{VI})$-induced mitochondria-dependent apoptosis. And activation of autophagy could repair mitochondria function to protect hepatocytes potentially by removing damaged mitochondria
\end{abstract}




\section{Introduction}

Chromium is ubiquitous in the environment, principally in trivalent and hexavalent forms [1]. While $\mathrm{Cr}(\mathrm{III})$ is an essential micronutrient for human, highly toxic $\mathrm{Cr}(\mathrm{VI})$ is widely used in many different industries including welding, chrome pigmenting and leather tanning. Occupational exposure to $\mathrm{Cr}(\mathrm{VI})$ compounds is associated with several adverse effects on human health, such as nasal perforation and bronchiogenic cancer. $\mathrm{Cr}(\mathrm{VI})$ has long been recognized as an inhalation carcinogen, according to the research that the accident of lung cancer in human and animals increased as a result of the exposure to $\operatorname{Cr}(\mathrm{VI})$ [2]. However, recent studies suggested ingestion carcinogenicity in animals and human when exposed to $\mathrm{Cr}(\mathrm{VI})$ in drinking water $[3,4]$, which derives comprehensive attention on the human ingestion toxicity of $\mathrm{Cr}(\mathrm{VI})$.

The liver is a critical organ to metabolize and detoxify exogenous toxicants in mammals and humans, which is easily attacked. Apoptosis is a conserved cellular suicide mechanism in which multiple signaling pathways are implicated. There have been described two major pathways of apoptosis: mitochondria-dependent or -independent. Our previous studies have demonstrated the ROS formation and mitochondria stress were the most typical events required for $\mathrm{Cr}(\mathrm{VI})$-induced apoptosis in L-02 hepatocytes [5]. When mitochondria are attacked by exogenous toxins, electron leaks from specific sites of mitochondrial electron transport chain (ETC) would be reduced to superoxide anion radical $\left(\mathrm{O}_{2}{ }^{\circ}\right)[6] . \mathrm{O}_{2}{ }^{-}$was further reduced to hydrogen peroxide $\left(\mathrm{H}_{2} \mathrm{O}_{2}\right)$ and hydroxyl radical $(\bullet \mathrm{OH}) \cdot \mathrm{O}_{2}^{--}, \mathrm{H}_{2} \mathrm{O}_{2}$ and $\bullet \mathrm{OH}$ are referred as ROS, collectively. Accumulating oxidative stresses inhibit mitochondrial complexes and affect the efficiency of mitochondria, and further increase the rate of ROS production. This results in several harmful changes, such as membrane permeability transition, ATP depletion and mitochondria dysfunction. These signals that converge on mitochondria would trigger the release of caspase activators (such as cytochrome $\mathrm{c}$ and AIF), participation of pro- and antiapoptotic Bcl-2 family proteins and further give rise to apoptotic cell death. Therefore, it is credible that removal and reduction of damaged mitochondria would be beneficial for $\mathrm{Cr}(\mathrm{VI})$-induced hepatotoxicity.

Macroautophagy (hereafter referred to as autophagy), is a cellular pathway by which cytoplasmic material and damaged organelles are degraded and recycled back as amino acid, nucleotide, fatty acid, and so on [7]. Autophagy occurs at low basal levels in all eukaryotes as a critical survival mechanism to maintain cellular homeostasis and energy balance as response to various death stimuli [8]. Under the regulation of series of autophagy-related genes (Atg), autophagy begins with the newly formation of double-membrane autophagosome, which enclose cellular content including lipid droplet and damaged organelles, thus degraded by the fusion with lysosomes via lysosomal hydrolase [9].

Although autophagy is usually activated as a survival mechanism in response to the pathogenesis of liver disease $[10,11]$, it is still unknown whether autophagy can modulate $\mathrm{Cr}(\mathrm{VI})$-induced hepatotoxicity. The present study were undertaken to explore the mechanism of $\mathrm{Cr}(\mathrm{VI})$-induced hepatotoxicity and evaluate the potentially protective effect of autophagy by the removal of damaged mitochondria.

\section{Materials and Methods}

Materials

Potassium dichromate $\left(\mathrm{K}_{2} \mathrm{Cr}_{2} \mathrm{O}_{7}\right)$, N-acetylcysteine (NAC), Chloroquine(CQ), Rapamycin (Rap) and 3-methyladenine (3-MA) were purchased from Sigma-Aldrich (St. Louis, MO, USA). RNA isolation and RTPCR reagents were obtained from Invitrogen (Carlsbad,CA, USA). All other chemicals and solvents were of analytical grade and obtained from Sigma-Aldrich except those specifically mentioned.

Human normal liver cell line (L-02) was obtained from the Cell Bank of the Chinese Academy of Sciences (Shanghai, China). All cells were cultured in RPMI-1640 medium supplemented with 10\% (vol/ 
Xie/Xiao/Luo/Zhong: Activation of Autophagy Protects Against $\mathrm{Cr}(\mathrm{VI})$-Induced Apoptosis in L-02 Hepatocytes

vol) $\mathrm{FBS}$ at $37^{\circ} \mathrm{C}$ in $5 \% \mathrm{CO}_{2}$. Protein concentrations were measured using a $\mathrm{BCA}$ protein quantification kit (Abcam, SF, USA) with bovine serum albumin used as concentration standards.

\section{Measurements of cell viability}

3-(4,5-dimethylthiazol- 2yl-)-2,5-diphenyl tetrazolium bromide (MTT) was used to evaluate viability of cells. L-02 hepatocytes were treated with $\mathrm{Cr}(\mathrm{VI})$ of different concentrations $(0-8 \mu \mathrm{M})$ for $24 \mathrm{~h}$. Then $10 \mu \mathrm{l}$ of a MTT solution (5 mg/ml in PBS as stock solution) was added into each well of 96-well plates, and incubated for additional $4 \mathrm{~h}$ at $37^{\circ} \mathrm{C}$. Cell viability was measured by treating with dimethylsulfoxide (DMSO) prior to reading at $492 \mathrm{~nm}$ with microplate reader (Powerwave XS2, Biotek, Vermont).

\section{Detection of mitochondria membrane potential}

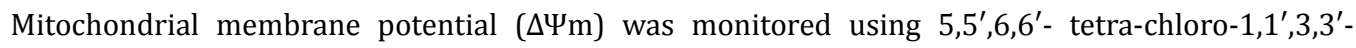
tetraethylbenzimidazolyl-carbocyanine iodide (JC-1), a lipophilic cationic fluorescence dye. JC-1 is capable of selectively entering mitochondria, where it forms monomers, and emits green fluorescence when $\Delta \Psi \mathrm{m}$ is relatively low. At a high $\Delta \Psi \mathrm{m}, \mathrm{JC}-1$ aggregates and gives red fluorescence. Thus the red and green fluorescence of JC- 1 reflect the change of $\Delta \Psi \mathrm{m}$ of the mitochondrial membrane. Briefly, cells were trypsinized, washed in ice-cold PBS, and incubated with JC-1 at $37^{\circ} \mathrm{C}$ for $20 \mathrm{~min}$. Finally, cells were washed twice with PBS and analyzed by FACScalibur flow cytometry (Becton Dickinson, NY, USA), measuring the fluorescence emission at $530 \mathrm{~nm}$ and excitation at $488 \mathrm{~nm}$.

\section{ATP content assay}

Cellular ATP content was assessed using the ATP assay kit (Abcam, Cambridge, UK) which utilizes the phosphorylation of glycerol to generate a product that is quantified by colorimetric methods. Cells were disposed under assay protocol and ATP content was measured under 570nm.

\section{Caspase 3 activity assay}

According to Abcam caspase-3 assay kit, caspase-3 activity assay is based on spectrophotometric detection of the chromophore p-nitronanilide (pNA), after its cleavage from the labeled substrate, acetylAsp-Glu-Val-Asp p-nitroanilide (Ac-DEVD-pNA). The activities of caspase-3 were described as the cleavage of the colorimetric substrate by measuring the absorbance at $405 \mathrm{~nm}$.

\section{Determination of apoptosis rate}

Apoptosis rates were detected with Invitrogen Annexin V/PI cell apoptosis kit. Harvest the cells after the incubation period and re-suspend the cells in Annexin-binding buffer. Add $5 \mu$ L FITC-labeled Annexin $\mathrm{V}$ and $1 \mu \mathrm{L} 100 \mu \mathrm{g} / \mathrm{mL}$ PI working solution to each $100 \mu \mathrm{L}$ of cell suspension. Then the apoptosis rates were calculated by the percentage of stained cells using FACSCAlibur flow cytometry, measuring the fluorescence emission at $530 \mathrm{~nm}$ and excitation at $488 \mathrm{~nm}$.

\section{Measurement of ROS production}

The fluorescent probe 5-(and 6)-chloromethyl-2',7'-dichlorodihydrofluorescein diacetate (CM$\mathrm{H}_{2}$ DCFDA, Molecular Probers, USA) are specific dyes used for ROS content. L-02 hepatocytes were seeded onto a glass coverslip in the bottom of a 6-well plate overnight. The cells were incubated with CM- $\mathrm{H}_{2}$ DCFDA ( $5 \mu \mathrm{M}$ final) for $30 \mathrm{~min}$. Cells were washed with PBS, mounted, and observed under Motican 3000 fluorescence microscope (Motic, Xiamen, China). In addition, for quantitative examination, cells were directly seeded into $60 \mathrm{~mm}$ culture dishes and treated with $\mathrm{CM}-\mathrm{H}_{2}$ DCFDA as described above. The fluorescence intensity was assessed by VarioskanFlash fluorescence microplate reader (Thermo Scientific, MA, USA) with excitation wavelength at $488 \mathrm{~nm}$ and emission wavelength at $525 \mathrm{~nm}$.

\section{Determination of $\mathrm{Ca}^{2+}$ content and $\mathrm{Ca}^{2+}$ channel activity}

L-02 hepatocytes were incubated at $5 \mu \mathrm{M}$ Fluo-3 AM (Beyotime, Nanjing, China) at $37^{\circ} \mathrm{C}$ for $30 \mathrm{~min}$. Then cells were harvested, washed three times with PBS and then re-suspended with RPMI-1640 medium. The fluorescence intensity was assessed by VarioskanFlash fluorescence microplate reader with excitation wavelength at $488 \mathrm{~nm}$ and emission wavelength at $525 \mathrm{~nm}$. Currents of calcium release activated channel (CRAC) were recorded at room temperature using a computer-based patch-clamp amplifier in whole- 
$\mathrm{Xie} /$ Xiao/Luo/Zhong: Activation of Autophagy Protects Against $\mathrm{Cr}(\mathrm{VI})$-Induced Apoptosis in L-02 Hepatocytes

Table 1. Primers used for semi-quantitative RT-PCR assays

\begin{tabular}{clc}
\hline Gene & \multicolumn{1}{c}{ Primer } & Product size \\
\hline \multirow{2}{*}{ Beclin1 } & Forward 5'- CCGCAAGATAGTGGCAGAA-3' & \\
& Reverse 5'- CGACCCAGCCTGAAGTTAT-3' & 264 bp \\
& Forward 5'- AGGGGCCGGACTCGTCATACT-3' & 202 bp \\
\multirow{2}{*}{ 3-actin } & \begin{tabular}{l} 
Reverse 5'- GGCGGCACCACCATGTACCCT-3' \\
\hline
\end{tabular}
\end{tabular}

cell patch clamping mode. Patch-pipettes were pulled (Narishige PC10) and fire-polished to 2-3M $\Omega$ (Narishige MF830). Recordings of membrane currents were made using a Multiclamp 700B amplifier (Axon Instruments) and a CV-70 headstage. The bath solution contained $140 \mathrm{mM} \mathrm{NaCl}, 2.8 \mathrm{mM} \mathrm{KCL}, 10 \mathrm{mM} \mathrm{CaCl}{ }_{2}$, $0.5 \mathrm{mM} \mathrm{MgCl}{ }_{2}, 11 \mathrm{mM}$ glucose, $10 \mathrm{mM}$ HEPES, adjusted to $\mathrm{pH} 7.4$ with $\mathrm{NaOH}$. The pipette solution contained $140 \mathrm{mM}$ K-glutamate, $8 \mathrm{mM} \mathrm{NaCl}, 1 \mathrm{mM} \mathrm{MgCl}{ }_{2}, 0.5 \mathrm{mM}$ Mg-ATP, $10 \mathrm{mM}$ EGTA, $10 \mathrm{mM} \mathrm{HEPES}$, adjusted to $\mathrm{pH} 7.2$ with $\mathrm{NaOH}$. To monitor changes in the amplitude of membrane currents, voltage ramps between $-100 \mathrm{mV}$ and $-80 \mathrm{mV}$ were applied every $2 \mathrm{~s}$, starting immediately after achieving the whole-cell configuration. Acquired currents were sampled at $10 \mathrm{kHz}$ and filtered at $2 \mathrm{kHz}$. Pipette and cell capacitance were compensated. Voltage-clamp commands were generated using Clampex 10.2 (Axon Instruments). Data were recorded via a Digidata 1440A interface (Axon Instruments).

\section{RNA isolation and RT-PCR analysis}

We assessed the induction of autophagy by determination the mRNA expression of Beclin 1. Specific primers for Beclin 1 and $\beta$-Actin were designed using Primer 5.0 software and are shown in Table 1. Total RNA was isolated from cells using Trizol reagent and spectrophotometrically quantified at 260nm. 260/280 ratio was also determined to confirm its quality. First strand cDNA was synthesized from total RNA using RevertAid First Strand cDNA Synthesis Kit (Thermo Scientific, NY, USA). The reaction was performed in thermocycler (Bio-Rad, CA, USA) at $42^{\circ} \mathrm{C}$ for $60 \mathrm{~min}$, and $70^{\circ} \mathrm{C}$ for $5 \mathrm{~min}$. Samples were thermocycled for PCR amplification (Roche, Mannheim, Germany). The reaction mixture contained $1 \mu \mathrm{L}$ cDNA, $10 \mu \mathrm{L} 2 \times$ SYBR Green qPCR Mix and $0.5 \mu \mathrm{L}$ of each primer $(5 \mu \mathrm{M})$ in a final volume of $20 \mu \mathrm{L}$. Reaction conditions for PCR were: $3 \mathrm{~min}$ at $95^{\circ} \mathrm{C}$ followed by $10 \mathrm{~s}$ at $95^{\circ} \mathrm{C}$ and $30 \mathrm{~s}$ at $58^{\circ} \mathrm{C}$. The optimum cycle number resulted to be 35 cycles for Beclin 1 and $\beta$-Actin.

\section{Western blot assay}

Cells were washed in PBS, and cell pellets were suspended in lysis buffer and centrifuged. Then $30 \mu \mathrm{g}$ of protein were separated by SDS-PAGE on $12 \%$ or $15 \%$ polyacrylamide gels and transferred to polyvinylidene difluoride membranes (PVDF). The membranes were incubated with 5\% non-fat dried milk for $1 \mathrm{~h}$ and incubated overnight at $4^{\circ} \mathrm{C}$ with anti-LC3 (1:1000 dilution; No. 128025; Abcam, SF, USA), anti-phospho-4EBP1(1:1000 dilution; No. 236B4; Cell Signaling, BSN, USA) and anti- $\beta$-Actin (1:3000; No. 4967; Cell Signaling, BSN, USA). Then membranes were incubated with anti-rabbit secondary antibodies and visualized using a chemiluminescent substrate (Thermo Scientific, MA, USA). All experiments were performed in at least triplicate.

\section{Observation of autophagosome}

To directly observe autophagosomes, hepatocytes were harvested and cell deposit were fixed overnight with $2.5 \%$ glutaraldehyde for $30 \mathrm{~min}$ at $4{ }^{\circ} \mathrm{C}$, then post-fixed with $2 \%$ osmic acid, dehydrated, and embedded with epoxy resin. Thin sections were observed with an H-600 transmission electron microscope (Hitachi, Tokyo, Japan).

\section{Mitochondria isolation}

Cells were harvested and resuspended in an ice-cold lysis buffer $(250 \mathrm{mM}$ sucrose, $20 \mathrm{mM}$ HEPES$\mathrm{KOH}, 10 \mathrm{mM} \mathrm{KCl}, 1.5 \mathrm{mM} \mathrm{MgCl}$, $1 \mathrm{mM}$ EGTA, $1 \mathrm{mM}$ EDTA, $1 \mathrm{mM}$ DTT and $0.1 \mathrm{mM}$ PMSF, pH 7.5). The cellular suspension is homogenized with a Teflon-glass homogenizer with 20 up and down passes of the pestle. The homogenate is then centrifuged at $750 \times \mathrm{g}$ for $10 \mathrm{~min}$, and the supernatants were further centrifuged at $10,000 \mathrm{~g}$ for $15 \mathrm{~min}$ at $4{ }^{\circ} \mathrm{C}$. The mitochondria pellet was washed once with lysis buffer and re-suspended 


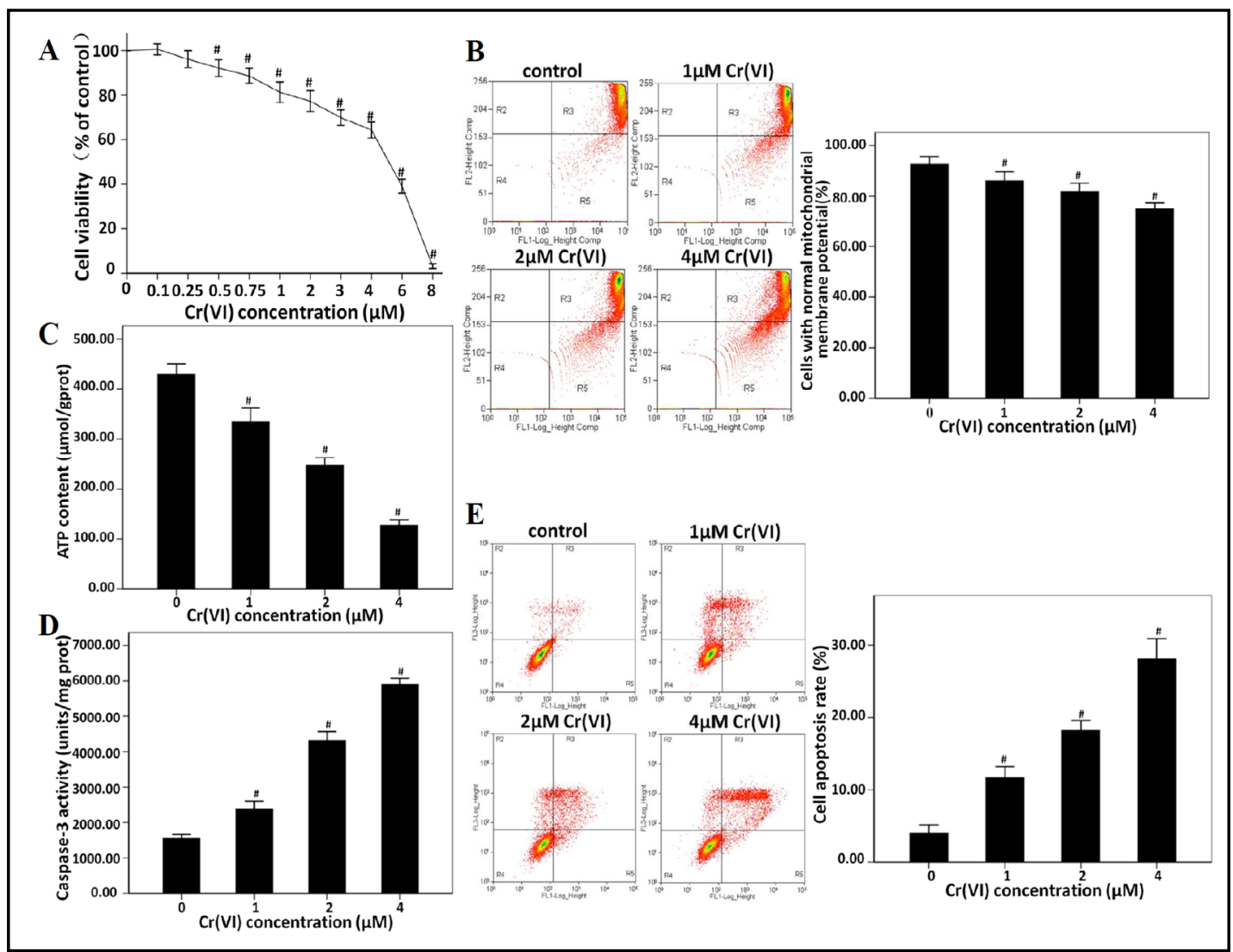

Fig. 1. $\mathrm{Cr}(\mathrm{VI})$ induces mitochondria-dependent apoptosis in L-02 hepatocytes. (A) L-02 hepatocytes were incubated with indicated concentrations for $24 \mathrm{~h}$ and then were subjected to a MTT assays for evaluating the percentage of viable cells. (B-C) After exposed to $\mathrm{Cr}(\mathrm{VI})$ with increasing concentrations $(0-4 \mu \mathrm{M})$ for $24 \mathrm{~h}$, mitochondrial membrane potential and ATP production were assessed in L-02 hepatocytes. (D-E) Cells were treated with $(0-4 \mu \mathrm{M}) \mathrm{Cr}(\mathrm{VI})$ for $24 \mathrm{~h}$, then caspase-3 activities and apoptosis rates of hepatocytes were detected with microplate reader and flow cytometry. The results are presented as mean $\pm \operatorname{SEM}(n=5)$. ${ }^{\#} P<0.05$ compared to $0 \mu \mathrm{M} \mathrm{Cr}(\mathrm{VI})$ group.

in lysis buffer. Isolated mitochondria were used immediately for measurement of mitochondrial respiration function.

\section{Measurement of mitochondrial respiration function}

Mitochondrial respiratory function was measured using a Clark oxygen electrode (Hansatech Instruments, Norfolk, UA) in a thermostat controlled chamber. Briefly, mitochondrial suspension (1mg protein) were added to $1 \mathrm{~mL}$ of respiratory medium preheated to $25^{\circ} \mathrm{C}\left(300 \mathrm{mM}\right.$ mannitol, $10 \mathrm{mM} \mathrm{NaH}_{2} \mathrm{PO}_{4}$, $5 \mathrm{mM} \mathrm{MgCl}, 10 \mathrm{mM} \mathrm{KCl}, \mathrm{PH}=7.2$ ). Mitochondrial respiration was initiated by addition of the complex II substrate succinate $(2.5 \mathrm{mM}$ final), and state 4 respiration was first measured in the absence of ADP for 2 mins. Subsequently, state 3 respiration was initiated by the addition of ADP (7.5mM final) to determine the maximal rate of coupled ATP synthesis. Respiration rate was measured by the slope of oxygen consumption curves and respiratory control ratio (RCR) was calculated as the ratio of state 3 to state 4 respiration rate.

\section{Statistical analysis}

All the data are expressed as mean \pm SEM. Comparisons among groups were performed with one-way ANOVA using SPSS 17.0 software or, where appropriate, by two-way ANOVA, followed by LSD test. A value of $P<0.05$ was considered statistically significant. 


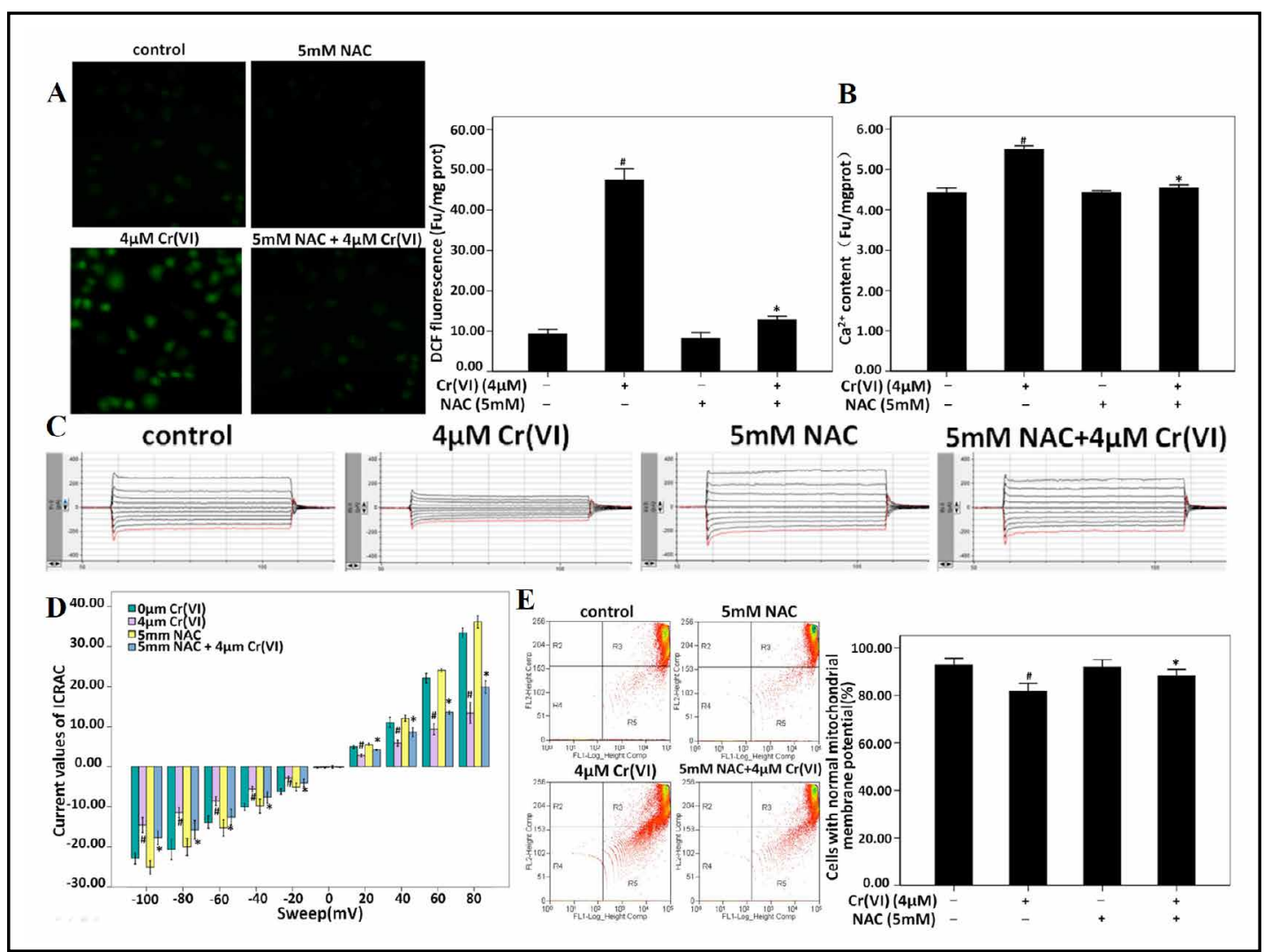

Fig. 2. ROS formation is a critical reason for mitochondrial damage in L-02 hepatocytes exposed to Cr(VI). (A) After L-02 hepatocytes treated with PBS and $4 \mu \mathrm{M} \mathrm{Cr}(\mathrm{VI})$ with or without $5 \mathrm{mM}$ NAC for $24 \mathrm{~h}$, ROS generation was detected with the fluorescence probe $\mathrm{CM}-\mathrm{H}_{2}$ DCFDA. (B) $\mathrm{Ca}^{2+}$ contents were assessed with probe Fluo-3 AM using fluorescence microplate reader. (C-D) CARC channel currents were detected with Multiclamp 700B patch-clamp amplifier for evaluating CRAC channel activities. (E) Mitochondrial membrane potential was detected with JC-1 staining. The results are shown as mean $\pm \mathrm{SEM}(\mathrm{n}=5) .{ }^{*} P<0.05$ compared with $0 \mu \mathrm{M}$ Cr(VI) group. ${ }^{*} P<0.05$ compared with $4 \mu \mathrm{M} \mathrm{Cr}(\mathrm{VI})$ group.

\section{Results}

Cr(VI) induces mitochondria-dependent apoptosis in L-02 hepatocytes in a dose-dependent manner

To determine the cytotoxic effects of $\mathrm{Cr}(\mathrm{VI})$ on L-02 hepatocytes, MTT assays were performed. As shown in Fig. 1A, the percentage of viable cells significantly diminished after treated with $0,0.1,0.25,0.5,0.75,1,2,3,4,6,8 \mu \mathrm{M} \mathrm{Cr}(\mathrm{VI})$ for $24 \mathrm{~h}$. Then cells were exposed to $\mathrm{Cr}(\mathrm{VI})$ of increasing concentrations $(1-4 \mu \mathrm{M})$ for $24 \mathrm{~h}$, and mitochondrial membrane potential and ATP production decreased in a concentration-dependent manner in Fig. 1B and C. Moreover, $\mathrm{Cr}(\mathrm{VI})$ also increased caspase- 3 activities and apoptosis rates increased significantly in Fig. 1D and E.

ROS formation is a critical reason for mitochondria damage in L-02 hepatocytes exposed to $\operatorname{Cr}(V I)$

ROS scavenger, $5 \mathrm{mM}$ NAC could effectively limit ROS production in hepatocytes treated with $4 \mu \mathrm{M} \mathrm{Cr}(\mathrm{VI})$ in Fig. $2 \mathrm{~A}$. To assess whether or not NAC may mitigate $\mathrm{Ca}^{2+}$ overload, we detected the $\mathrm{Ca}^{2+}$ contents and CARC channel activities. Results in Fig. 2B-D showed that $4 \mu \mathrm{M} \mathrm{Cr}(\mathrm{VI})$ increased $\mathrm{Ca}^{2+}$ contents and decreased CARC channel currents compared to control group, and the combination treatment of $\mathrm{NAC}$ and $\mathrm{Cr}(\mathrm{VI})$ inhibited $\mathrm{Ca}^{2+}$ overload and restored CARC channel activities. After application of $4 \mu \mathrm{M} \mathrm{Cr}(\mathrm{VI})$ and $5 \mathrm{mM}$ NAC for 


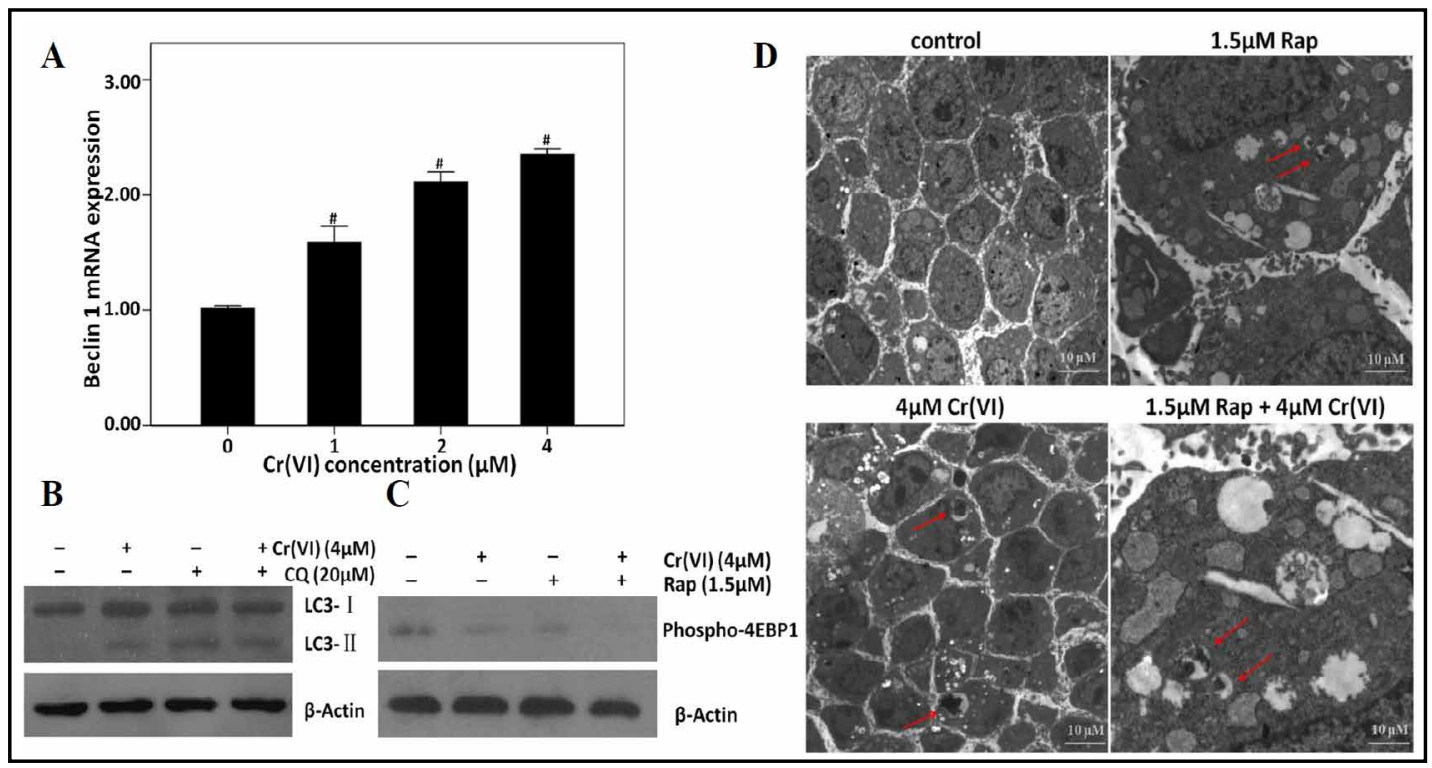

Fig. 3. $\mathrm{Cr}(\mathrm{VI})$ induces autophagy in L-02 hepatocytes. (A) Cells were treated with $\mathrm{Cr}(\mathrm{VI})$ with increasing concentrations (0-4 $\mu \mathrm{M})$ for $24 \mathrm{~h}$, then Beclin 1 mRNA expressions were assessed with RT-PCR. (B) Cells were treated with PBS, $\mathrm{Cr}(\mathrm{VI})(4 \mu \mathrm{M}), \mathrm{CQ}(20 \mu \mathrm{M})$ alone or $\mathrm{Cr}(\mathrm{VI})$ plus CQ for $24 \mathrm{~h}$. Total lysates were subjected to immunoblotting assay for LC3. (C-D) L-02 hepatocytes were exposed to PBS, $4 \mu \mathrm{M}$ Cr(VI), $1.5 \mu \mathrm{M}$ Rap or $4 \mu \mathrm{M}$ $\mathrm{Cr}(\mathrm{VI})+1.5 \mu \mathrm{M}$ Rap respectively. The phosphorylation level of mTOR downstream protein, translational initiation factor $4 \mathrm{E}$ binding protein-1 (4EBP-1) was detected by western blot assay. And cells were processed for EM analysis and representative images of autophagosomes were observed. Arrows denote autophagosomes containing damaged mitochondria. The results are presented as mean $\pm \mathrm{SEM}(\mathrm{n}=5)$. ${ }^{\#} \mathrm{P}<0.05$ compared with control group.

24h, mitochondria membrane potential increased compared to $4 \mu \mathrm{M} \mathrm{Cr}(\mathrm{VI})$ group in Fig. 2E. Therefore, these data revealed that NAC exerted an protective effect on $\mathrm{Cr}(\mathrm{VI})$-induced mitochondrial damage.

\section{Cr(VI) induces autophagy in L-02 hepatocytes}

As shown in Fig. 3A, Cr(VI) induced the increase of Beclin 1 mRNA expression compared to control group in a concentration-dependent manner. Autophagy flux was also assessed by using a combination of lysosomal inhibitor CQ in Fig. 3B. Cr(VI) treatment increased endogenous LC3-II levels and combined CQ and Cr(VI) treatement further increased the level of LC3-II protein. The phosphorylation level of mTOR downstream protein, translational initiation factor $4 \mathrm{E}$ binding protein-1 (4EBP-1) decreased in $\mathrm{Cr}(\mathrm{VI})$-induced hepatocytes in Fig. 3C. And combined $\mathrm{Cr}(\mathrm{VI})$ and mTOR inhibitor rapamycin treatment further decreased the phospho-4EBP1 protein levels. EM analysis indicated an increased accumulation of autophagosomes after $4 \mu \mathrm{M} \mathrm{Cr}(\mathrm{VI})$ and/or $1.5 \mu \mathrm{M}$ Rap treatment in Fig. 3D. It is noteworthy that the double-membrane autophagosomes had enveloped injured mitochondria, suggesting that autophagy may help remove the damaged mitochondria induced by $\mathrm{Cr}(\mathrm{VI})$.

\section{Activation of autophagy protects against Cr(VI)-induced apoptosis}

Cells were incubated with PBS, $4 \mu \mathrm{M} \mathrm{Cr}(\mathrm{VI})$ only, or co-treated with $1.5 \mu \mathrm{M}$ Rap or $5 \mathrm{mM}$ 3-MA for 24h. As shown in Fig. 4A, 1.5 $\mathrm{M}$ Rap ameliorated the decrease of mitochondria membrane potential caused by $\mathrm{Cr}(\mathrm{VI})$, which is deteriorated by autophagy inhibitor 3-MA $(5 \mathrm{mM})$. The mitochondria isolated from L-02 hepatocytes co-treated with Rap exhibited higher RCR compared to $4 \mu \mathrm{M} \mathrm{Cr}(\mathrm{VI})$ group, while $5 \mathrm{mM} 3-\mathrm{MA}$ aggravated mitochondria 


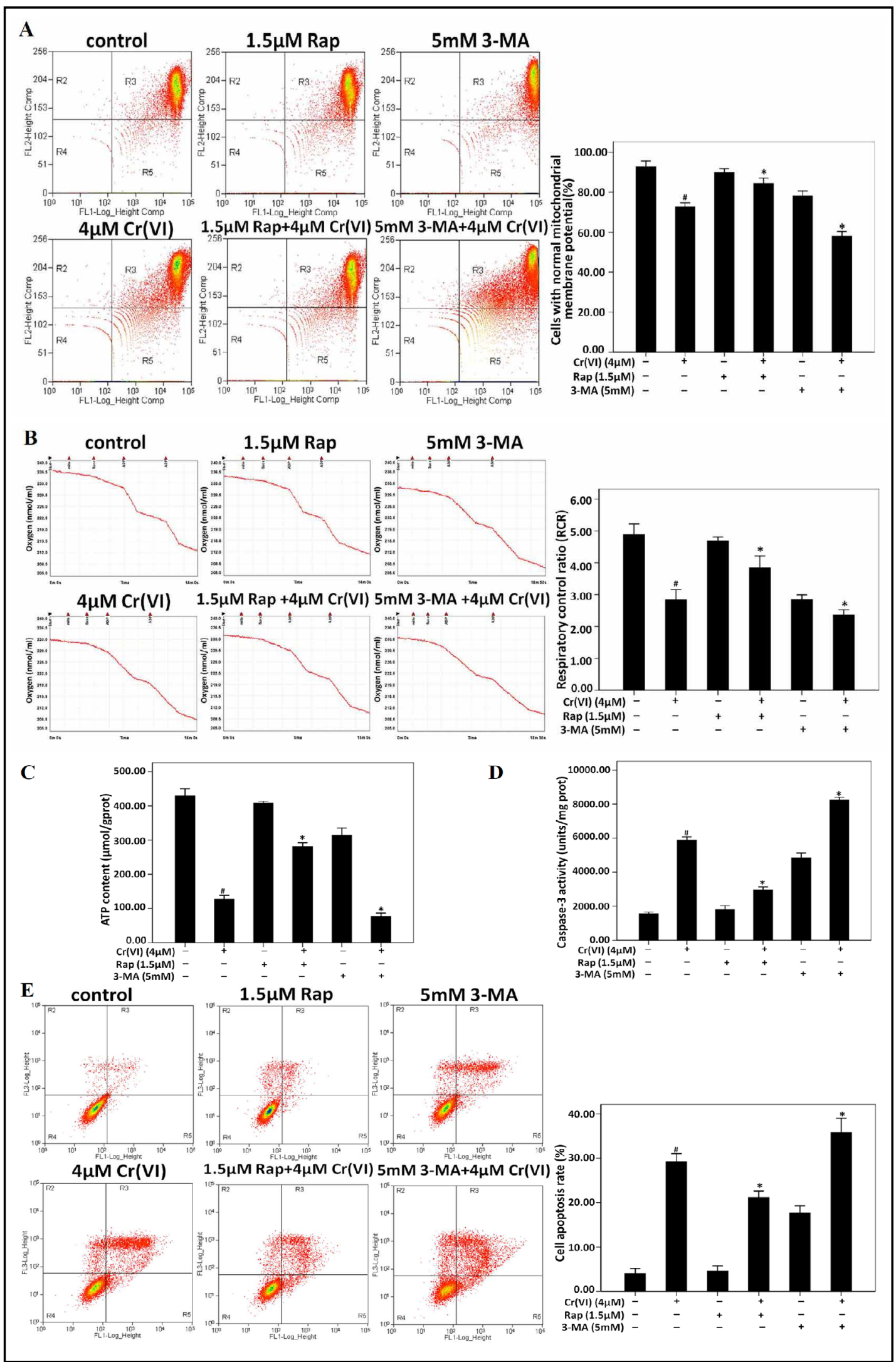

Fig. 4. Activation of autophagy protects against $\mathrm{Cr}(\mathrm{VI})$-induced hepatocelluar apoptosis. Administration of autophagy inducer Rap and autophagy inhibitor 3-MA modulated Cr(VI)-induced apoptosis in L-02 he- 
patocytes. L-02 hepatocytes were divided into 6 groups and treated with $\mathrm{PBS}, 4 \mu \mathrm{M} \mathrm{Cr}(\mathrm{VI})$ only, or co-treated with $1.5 \mu \mathrm{M}$ Rap or 5mM 3-MA for 24h. (A) Mitochondria membrane potential was detected with JC-1 staining. (B-C) Mitochondria respiratory curves were recorded by Clark oxygen electrode and respiratory control ratio (RCR) were calculated. (D-E) The caspase-3 activities and apoptosis rates of hepatocytes were detected with microplate reader and flow cytometry. The results are shown as mean \pm SEM $(n=5) .{ }^{*} P<0.05$ compared with control group. ${ }^{*} P<0.05$ compared with $4 \mu \mathrm{M} \mathrm{Cr}(\mathrm{VI})$ group.

respiratory function in Fig. 4B. Due to that mitochondria normal respiratory function is essential for ATP production, $\mathrm{Cr}(\mathrm{VI})$-induced the reduction of ATP generation could be recovered to a higher level after co-treated with Rap, while 3-MA aggravated mitochondrial imbalance of energy metabolism in Fig. 4C. Finally, caspase-3 activities and apoptosis rates decreased in $4 \mu \mathrm{M} \mathrm{Cr}(\mathrm{VI})+1.5 \mu \mathrm{M}$ Rap group compared to $4 \mu \mathrm{M} \mathrm{Cr}(\mathrm{VI})$ group, and in contrast, caspase- 3 activities and apoptosis rates in $5 \mathrm{mM} 3-\mathrm{MA}+4 \mu \mathrm{M} \mathrm{Cr}(\mathrm{VI})$ group were significantly higher than that in $4 \mu \mathrm{M} \mathrm{Cr}(\mathrm{VI})$ group in Fig. $4 \mathrm{D}$ and $\mathrm{E}$.

\section{Discussion}

Mitochondria are critical for cellular energy metabolism and cellular homeostasis. The study found that $\mathrm{Cr}(\mathrm{VI})$ induced the decrease of mitochondrial membrane potential in a concentration-dependent manner. Mitochondria permeability transition (MPT) would cause the release of cytochrome c (Cyt c). Cyt c, as a water-soluble electron carrier is indispensable for oxidative phosphorylation. Cyt c passes electron to cytochrome c oxidase, which uses the electrons and hydrogen ions to reduce molecular oxygen to water. So Cyt c release aggravated the blockage of mitochondria electron transport chain and diminished mitochondrial ATP production. And the release of cytochrome c from mitochondria to cytoplasm brought about caspase activation, which is an important step in programmed cell death. Our study has demonstrated that $\mathrm{Cr}(\mathrm{VI})$ induced cell death of L-02 hepatocytes through mitochondriamediated and caspase-dependent pathways.

Considering that excess ROS generation would be upstream signaling of mitochondria damage, NAC as a antioxidant were used to assess the role of ROS in mitochondria injury induced by $\mathrm{Cr}(\mathrm{VI})$. Mitochondria are the main sources of ROS, and therefore are vulnerable to ROS damage. ROS directly attacks mitochondrial respiratory chain enzymes, or by the induction of mutations of the mitochondrial genome, leading to the inhibition of mitochondrial respiratory chain enzymes and the dysfunction of oxidative phosphorylation. The results have demonstrated that NAC diminished ROS formation to maintain mitochondria homeostasis.

ROS production is also related with calcium overload in hepatocytes, which could exacerbate mitochondria damage [12]. $\mathrm{Ca}^{2+}$ can enhance cytochrome c dislocation from the mitochondrial inner membrane, either by competing for cardiolipin binding sites or by inducing MPT, which results in an effective blockage of electron transport and enhancement of ROS generation [13]. Conversely, cytochrome c can bind to the endoplasmic reticulum inositol 1,4,5-trisphosphate receptor (IP3R), resulting in enhanced ER $\mathrm{Ca}^{2+}$ release and further $\mathrm{Ca}^{2+}$ overload [14]. According to our results, NAC inhibits the increase of $\mathrm{Ca}^{2+}$ content in L-02 hepatocytes exposed to $\mathrm{Cr}(\mathrm{VI})$. Calcium release activated channels (CRAC) are specialized plasma membrane $\mathrm{Ca}^{2+}$ ion channels in hepatocytes [15]. $\mathrm{Cr}(\mathrm{VI})$ decreased the current amplitude of CRAC, while co-treatment with NAC could restore CRAC activities. Therefore, NAC, as a wildly used antioxidant, repaired mitochondrial dysfunction by the clearance of ROS generation and the suppression of calcium overload.

Many mitochondrial toxins regulate autophagic process [16]. Both mitochondrial superoxide and MPT are reported to influences autophagic flux $[17,18]$. Beclin 1 , mammalian homolog of Atg6, is reported to be essential for autophagosome formation [19]. We found that $\mathrm{Cr}(\mathrm{VI})$ increased the mRNA expression of pro-autophagic protein Beclin 1 in L-02 
hepatocytes. Light chain 3 (LC3), a mammalian homolog of Atg8, is a critical protein in autophagic process. The phosphatidylethanolamine-conjugated form of LC3-II translocates to the autophagosomal membrane and promotes the formation of a double-membrane autophagosome, so the increase of LC3-II levels is a landmark change to verify autophagic flux. We treated hepatocytes with $\mathrm{Cr}(\mathrm{VI})$ in the absence or presence of lysosomal inhibitor chloroqiune (CQ). CQ, a commonly used antimalarial drug, increases lysosomal pH and blocks the fusion of autophagosome with lysosomes, and then autophagic protein LC3-II degradation was inhibited [20]. The results showed that Cr(VI) induced the increase of LC3-II levels and combined CQ and $\mathrm{Cr}(\mathrm{VI})$ treatement further increase the level of LC3-II protein. In addition, under stress conditions, suppression of mammalian target of rapamycin (mTOR) is a central molecular signaling pathway leading to autophagy induction [10], and we further found that $\mathrm{Cr}(\mathrm{VI})$ treatment would decreased the level of phosphorylated 4EBP1, a downstream target protein of mTOR in L-02 hepatocytes. Another criterion for autophagy is to monitor the formation of autophagosome [21]. The study confirmed autophagic degradation of damaged mitochondria by observing double-membrane mitochondria-containing vacuolar structures using transmission electron microscopy [22]. All these results supported that $\mathrm{Cr}(\mathrm{VI})$ induced autophagy in hepatocytes. It could be suggested that autophagy act as a survival mechanism in trying to rescue cells from apoptosis, but cell fate may be finally as a result of a balance between cell rescue and cell damage. Recent studies suggested that autophagy reduces alcohol-induced liver injury by the removal of damaged mitochondria [23]. When $\mathrm{Cr}(\mathrm{VI})$-activated autophagy by itself could not resist cellular damage, selective autophagic elimination of depolarized mitochondria might be a feasible way to eliminate defective mitochondria and augment energy production.

Mitochondria are common targets for various toxicants-induced hepatotoxicities, and maintaining a healthy population of mitochondria is necessary for the well-being of hepatocytes. Numerous studies have demonstrated that autophagy could mitigate hepatocytes injuries by selectively removing damaged mitochondria [24]. Several signaling pathways are involved to regulate autophagy in mammalians cells. Rapamycin, as an mTOR inhibitor, could be used as a standard inducer for autophagy as stated above [25]. Another critical regulator of autophagy is the class III PI3k Vps34, which is necessary for the formation of autophagosomes, so pharmacological inhibition of autophagy by 3-MA leads to inhibition of autophagosome formation [26]. We investigated the effects of rapamycin or 3-MA on L-02 hepatocytes cell death caused by $\mathrm{Cr}(\mathrm{VI})$.

Mitochondrial oxidative phosphorylation is the major ATP synthetic pathway in hepatocytes. Eliminate damaged mitochondria to avoid vicious cycle of excess ROS generation and impaired respiratory function is beneficial to stimulate ATP synthesis and maintain mitochondrial homeostasis [27]. Our studies demonstrated that pretreatment with rapamycin prevented the reduction of the mitochondrial membrane potential, and then restoring mitochondrial respiration function and ATP content. Rapamycin also results in lower levels of cytochrome c release into the cytosol by the induction of Bcl-2 protein levels [28]. Apoptosis is an energy-dependent process that is regulated by a cascade activation of caspase proteins. Several studies found that autophagy inhibitors increased DNA fragmentation, caspase-3 activation and apoptosis [29]. Consistent with these findings, pharmacological suppression of autophagy by 3-MA accelerated apoptotic cell death in L-02 hepatocytes exposed to $\mathrm{Cr}(\mathrm{VI})$. Co-treatment $\mathrm{Cr}(\mathrm{VI})$ with 3-MA markedly increased mitochondria damage, caspase activation and ensuing apoptotic cell death in our experiments. Therefore, the activation of autophagy was beneficial for restoring mitochondria biogenesis and maintaining mitochondrial cellular homeostasis probably by selective elimination of mitochondria [30].

In conclusion, we have demonstrated that $\mathrm{Cr}(\mathrm{VI})$ induces ROS-mediated mitochondriadependent apoptosis in L-02 hepatocytes. NAC exhibited a protective role against ROS accumulation and mitochondria damage. Moreover, the present study for the first time demonstrated the activation of autophagy apparently attenuated $\operatorname{Cr}(\mathrm{VI})$-induced hepatotoxicity and the protection may be mediated by the removal of damaged mitochondria. 
Water contaminated with $\mathrm{Cr}(\mathrm{VI})$ has been suggested as a severe health threat in humans through oral route. These finding implied that induction of autophagy could be a novel therapeutic approach to mitigate $\mathrm{Cr}(\mathrm{VI})$-induced hepatotoxicity and liver injury and protect human health.

\section{Acknowledgments}

We are very grateful for the generous assistance of Evaluation and Research Center for Toxicology, Institute of Disease Control and Prevention, Academy of Military Medical Sciences, Beijing, China. This work was supported by the grants from Chinese National Natural Sciences Foundation (No. 81172701) and Hunan Provincial Innovation Foundation For Postgraduate (NO. 71380100001). And the authors declare no conflict of interest.

\section{References}

1 Quinteros FA, Machiavelli LI, Miler EA, Cabilla JP, Duvilanski BH: Mechanisms of chromium (VI)-induced apoptosis in anterior pituitary cells. Toxicology 2008;249:109-115.

-2 Seidler A, Jähnichen S, Hegewald J, Fishta A, Krug O, Rüter L, Strik C, Hallier E, Straube S: Systematic review and quantification of respiratory cancer risk for occupational exposure to hexavalent chromium. Int Arch Occup Environ Health 2013;86:943-955.

-3 Stern AH: A quantitative assessment of the carcinogenicity of hexavalent chromium by the oral route and its relevance to human exposure. Environ Res 2010;110:798-807.

-4 Linos A, Petralias A, Christophi CA, Christoforidou E, Kouroutou P, Stoltidis M, Veloudaki A, Tzala E, Makris $\mathrm{KC}$, Karagas MR: Oral ingestion of hexavalent chromium through drinking water and cancer mortality in an industrial area of Greece--an ecological study. Environ Health 2011;10:50.

-5 Xiao F, Li Y, Dai L, Deng Y, Zou Y, Li P, Yang Y, Zhong C: Hexavalent chromium targets mitochondrial respiratory chain complex I to induce reactive oxygen species-dependent caspase-3 activation in L-02 hepatocytes. Int J Mol Med 2012;30:629-635.

6 Murphy MP: How mitochondria produce reactive oxygen species. Biochem J 2009;417:1-13.

7 Mizushima N, Levine B, Cuervo AM, Klionsky DJ: Autophagy fights disease through cellular self-digestion. Nature 2008;451:1069-1075.

8 Ni HM, Williams JA, Yang H, Shi YH, Fan J, Ding WX: Targeting autophagy for the treatment of liver diseases. Pharmacol Res 2012;66:463-474.

9 Mehrpour M, Esclatine A, Beau I, Codogno P: Overview of macroautophagy regulation in mammalian cells. Cell Res 2010;20:748-762.

10 Ni HM, Bockus A, Boggess N, Jaeschke H, Ding WX: Activation of autophagy protects against acetaminophen-induced hepatotoxicity. Hepatology 2012;55:222-232.

11 Cardinal J, Pan P, Tsung A: Protective role of cisplatin in ischemic liver injury through induction of autophagy. Autophagy 2009;5:1211-1212.

12 Petrosillo G, Ruggiero FM, Pistolese M, Paradies G: Ca2+-induced reactive oxygen species production promotes cytochrome $\mathrm{c}$ release from rat liver mitochondria via mitochondrial permeability transition (MPT)-dependent and MPT-independent mechanisms: role of cardiolipin. J Biol Chem 2004;279:5310353108.

13 Iverson SL, Orrenius S: The cardiolipin-cytochrome c interaction and the mitochondrial regulation of apoptosis. Arch Biochem Biophys 2004;423:37-46.

14 Brookes PS, Yoon Y, Robotham JL, Anders MW, Sheu SS: Calcium, ATP, and ROS: a mitochondrial love-hate triangle. Am J Physiol Cell Physiol 2004;287: 817-833.

15 Ferreira R, Schlichter LC: Selective activation of KCa3.1 and CRAC channels by P2Y2 receptors promotes $\mathrm{Ca}^{2+}$ signaling, store refilling and migration of rat microglial cells. PLoS One 2013;8:e62345.

16 Chen Y, Gibson SB: Is mitochondrial generation of reactive oxygen species a trigger for autophagy? Autophagy 2008:4:246-248. 


\section{Cellular Physiology $\quad$ Cell Physiol Biochem 2014;33:705-716 \begin{tabular}{l|l} 
and BiOChemistry & $\begin{array}{l}\text { DOI: 10.1159/000358646 } \\
\text { Published onlIne: VIarch 07, 2014 }\end{array}$ \\
\hline
\end{tabular} \\ Xie/Xiao/Luo/Zhong: Activation of Autophagy Protects Against Cr(VI)-Induced Apoptosis in L-02 Hepatocytes}

17 Kim EH, Choi KS: A critical role of superoxide anion in selenite-induced mitophagic cell death. Autophagy 2008;4:76-78.

18 Ding WX: Role of autophagy in liver physiology and pathophysiology. World J Biol Chem 2010:1:3-12.

19 Rana RM, Dong S, Ali Z, Huang J, Zhang HS: Regulation of ATG6/Beclin-1 homologs by abiotic stresses and hormones in rice (Oryza sativa L.). Genet Mol Res 2012;11:3676-3687.

-20 Mei S, Ni HM, Manley S, Bockus A, Kassel KM, Luyendyk JP, Copple Bl, DingWX. Differential roles of unsaturated and saturated fatty acids on autophagy and apoptosis in hepatocytes. J Pharmacol Exp Ther 2011:339:487-498.

-21 Tanida I: Autophagosome formation and molecular mechanism of autophagy. Antioxid Redox Signal 2011;14:2201-2214.

-22 Apostolova N, Gomez-Sucerquia LJ, Gortat A, Blas-Garcia A, Esplugues JV: Autophagy as a rescue mechanism in efavirenz-induced mitochondrial dysfunction: a lesson from hepatic cells. Autophagy 2011;7:1402-1404.

23 Ding WX, Li M, Chen X, Ni HM, Lin CW, Gao W, Lu B, Stolz DB, Clemens DL, Yin XM: Autophagy reduces acute ethanol-induced hepatotoxicity and steatosis in mice. Gastroenterology 2010;139:1740-1752.

24 Kim I, Rodriguez-Enriquez S, Lemasters JJ: Selective degradation of mitochondria by mitophagy. Arch Biochem Biophys 2007;462:245-253.

25 Ravikumar B, Sarkar S, Davies JE, Futter M, Garcia-Arencibia M, Green-Thompson ZW, Jimenez-Sanchez M, Korolchuk VI, Lichtenberg M, Luo S, Massey DC, Menzies FM, Moreau K, Narayanan U, Renna M, Siddiqi FH, Underwood BR, Winslow AR, Rubinsztein DC: Regulation of mammalian autophagy in physiology and pathophysiology. Physiol Rev 2010;90:1383-1435.

26 Jaber N, Dou Z, Chen JS, Catanzaro J, Jiang YP, Ballou LM, Selinger E, Ouyang X, Lin RZ, Zhang J, Zong WX: Class III PI3K Vps34 plays an essential role in autophagy and in heart and liver function. Proc Natl Acad Sci USA 2012;109:2003-2008.

27 Novak I: Mitophagy: a complex mechanism of mitochondrial removal. Antioxid Redox Signal 2012;17:794802.

-28 Park JH, Lee JE, Lee SJ, Park SJ, Park KH, Jeong M, Koh HC: Potential autophagy enhancers protect against fipronil-induced apoptosis in SH-SY5Y cells. Toxicol Lett 2013;223:25-34.

29 Vucicevic L, Misirkic M, Janjetovic K, Vilimanovich U, Sudar E, Isenovic E, Prica M, Harhaji-Trajkovic L, Kravic-Stevovic T, Bumbasirevic V, Trajkovic V: Compound C induces protective autophagy in cancer cells through AMPK inhibition-independent blockade of Akt/mTOR pathway. Autophagy 2011;7:40-50.

-30 Youle RJ, Narendra DP: Mechanisms of mitophagy. Nat Rev Mol Cell Biol 2011;12:9-14. 